\title{
Traffic Pattern based Performance Comparison of Two Proactive MANET Routing Protocols using Manhattan Grid Mobility Model
}

\author{
Sunil Kr. Maakar \\ Research Scholar \\ Dept. of Computer Science \& Engg. \\ Punjab Technical University, India
}

\author{
Yudhvir Singh, Ph.D \\ Associate Professor \\ Dept. of Computer Science \& Engg. \\ M.D. University, India
}

\begin{abstract}
Mobile ad-hoc network is a collection of wireless mobile hosts forming a temporary network without the aid of any stand-alone infrastructure or centralized administration. Mobile Ad-hoc networks are self-organizing and self configuring multihop wireless networks where, the structure of the network changes dynamically. This is mainly due to the mobility of the nodes. In the current study we have compared the performance of three MANET routing protocols AODV as reactive, DSDV and OLSR as proactive by using Manhattan Grid mobility model. These share some similar behavior, but the protocols internal mechanism leads to significant performance difference. We have analyzed the performance of protocols by varying network load, mobility and type of traffic (CBR and TCP). Manhattan Grid Mobility model has been generated by IMPORTANT (Impact of Mobility Patterns on Routing in Ad-hoc NeTwork) tool. A detailed simulation has been carried out in NS2. The metrics used for performance analysis are Packet Delivery Fraction, Average end-toend Delay, Packet Loss, Routing Overhead, Normalized Routing Load and throughput. It has been observed that AODV (reactive) protocol performs better in CBR traffic and in case of real time delivery of packets but at cost of higher routing overhead. But in TCP traffic, proactive routing protocol OLSR gives better result. Overall performance of routing protocols in TCP traffic is much better than CBR traffic.
\end{abstract}

\section{Keywords}

MANET, Routing Protocol, IMPORTANT, TCP, CBR, Manhattan Grid Mobility Model, Performance Metrics

\section{INTRODUCTION}

Mobile networks can be classified into infrastructure networks and mobile ad hoc networks (MANET) according to their dependence on fixed infrastructures [2]. In an infrastructure mobile network, mobile nodes have wired access points (or base stations) within their transmission range. In contrast, mobile ad hoc networks are autonomously self-organized networks without infrastructure support. In a mobile ad hoc network, nodes move arbitrarily, therefore the network may experience rapid and unpredictable topology changes. Routing paths in MANETs potentially contain multiple hops, and every node in MANET has the responsibility to act as a router [4]. Routing in MANET has been a challenging task ever since the wireless networks came into existence. The major reason for this is the constant change in network topology because of high degree of node mobility. A number of protocols have been developed to accomplish this task. There exists various mobility models such as random way point, reference point group mobility model (RPGM), Manhattan mobility model, freeway mobility model, Gauss Markov mobility model etc that have been proposed for evaluation $[8$, 15].

\section{RELATED WORK}

Several performance evaluation of MANET routing protocols using CBR traffic have been done by considering various parameters such as mobility, network load and pause time. G. Jayakumar and G. Gopinath et. al. [19] investigated performance of AODV and DSR using Manhattan Grid Mobility Model with CBR traffic sources. They investigated that AODV perform better than DSR. Nor Surayati Mohamad Usop, Azizol Abdula and Ahmad [20] investigated the performance of AODV, DSDV and DSR in Grid Environment using CBR traffic. They concluded that AODV and DSDV performed better than DSR. Biradar, S. R. et al.[13] have analyzed the AODV and DSR protocol using Group Mobility Model and CBR traffic sources. Biradar, S. R. et. al.[13] investigated that DSR performs better in high mobility and average delay is better in case of AODV for increased number of groups. Also Rathy, R.K. et. al.[10] investigated AODV and DSR routing protocols under Random Way Point Mobility Model with TCP and CBR traffic sources. They concluded that AODV outperforms DSR in high load and/or high mobility situations.

In this paper we have investigated the performance of AODV (reactive), DSDV and OLSR (proactive) routing protocol for performance comparison in the scenario of movement of nodes in urban area (only in horizontal and vertical streets) . For this scenario we have used Manhattan Grid (MH) mobility model. The purpose of this work is to understand the working mechanism of protocols, Manhattan Grid Mobility Model and which routing protocol gives better performance in which situation or traffic when the nodes move only in horizontal or vertical streets.

The rest of the paper is organized as follows. In the next section we discuss the Manhattan Grid Mobility Model (MH). In section 4, we have given the brief introduction of AODV, DSDV and OLSR routing protocol. Section 5 and 6 deals the simulation setup and results obtained on the execution of simulation. Finally, we draw the conclusion in section 7.

\section{MANHATTAN GRID (MH) MOBILITY MODEL}

Fan Bai and Ahmed introduced the Manhattan model to emulate the movement pattern of mobile nodes on streets [7]. It can be useful in modeling movement in an urban area where a pervasive computing service between portable devices is provided. The scenario is composed of a number of horizontal and vertical streets. Given below is example topography showing the movement of nodes for Manhattan Mobility 
Model with seventeen nodes. The map defines the roads along which the nodes can move.

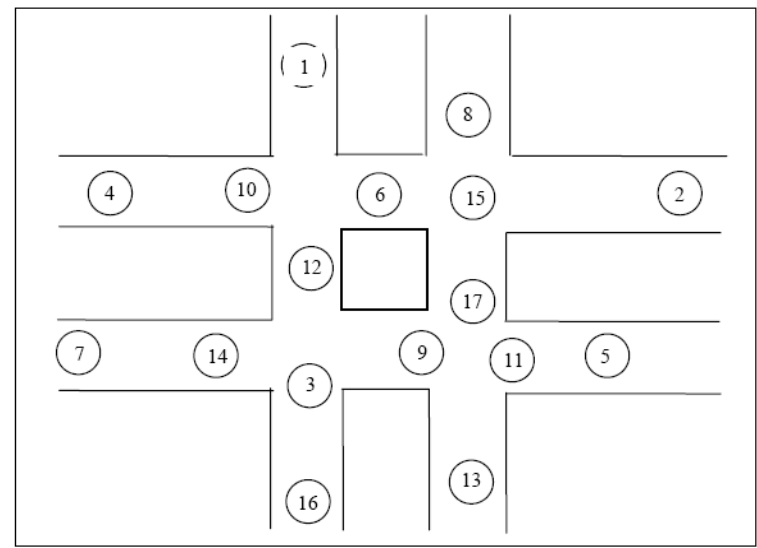

Figure 1: Movement of nodes for Manhattan Grid Mobility Model

Applications: It can be useful in modeling movement in an urban area where a pervasive computing service between portable devices is provided.

Important Characteristics: Maps are used in this model. However, the map is composed of a number of horizontal and vertical streets. Each street has two lanes for each direction (North and South direction for vertical streets, East and West for horizontal streets). The mobile node is allowed to move along the grid of horizontal and vertical streets on the map. At an intersection of a horizontal and a vertical street, the mobile node can turn left, right or go straight with certain probability. Except the above difference, the inter-node and intra-node relationships involved in the Manhattan model are very similar to the Freeway model.

Thus, the Manhattan mobility model is also expected to have high spatial dependence and high temporal dependence. It too imposes geographic restrictions on node mobility. However, it differs from the Freeway model in giving a node some freedom to change its direction.

\section{DESCRIPTION OF ROUTING PROTOCOL}

\subsection{Ad-Hoc on Demand Distance Vector (AODV)}

The Ad-hoc On-demand Distance Vector routing protocol $[1,3,14]$ enables multihop routing between the participating mobile nodes wishing to establish and maintain an ad-hoc network. AODV is a reactive protocol based upon the distance vector algorithm.

The algorithm uses different messages to discover and maintain links. Whenever a node wants to try and find a route to another node it broadcasts a Route Request (RREQ) to all its neighbors. The RREQ propagates through the network until it reaches the destination or the node with a fresh enough route to the destination. Then the route is made available by uncasing a RREP back to the source.

The algorithm uses hello messages (a special RREP) that are broadcasted periodically to the immediate neighbors. These hello messages are local advertisements for the continued presence of the node, and neighbors using routes through the broadcasting node will continue to mark the routes as valid. If hello messages stop coming from a particular node, the neighbor can assume that the node has moved away and mark that link to the node as broken and notify the affected set of nodes by sending a link failure notification (a special RREP) to that set of nodes.

\subsection{Destination Sequenced Distance Vector (DSDV)}

This routing protocol was developed 1994 by C. Perkins and it is a proactive distance-vector protocol $[4,9,15]$.

Destination-Sequenced Distance-Vector Routing protocol is a proactive table driven algorithm based on classic BellmanFord routing. In proactive protocols, all nodes learn the network topology before a forward request comes in. In DSDV protocol each node maintains routing information for all known destinations. The routing information is updated periodically. Each node maintains a table, which contains information for all available destinations, the next node to reach the destination, number of hops to reach the destination and sequence number. The nodes periodically send this table to all neighbors to maintain the topology, which adds to the network overhead. Each entry in the routing table is marked with a sequence number assigned by the destination node. The sequence numbers enable the mobile nodes to distinguish stale routes from new ones, thereby avoiding the formation of routing loops.

\subsection{Optimized Link State Routing Protocol (OLSR)}

Optimized Link state Routing protocol (OLSR) [4,11], is one of the proactive routing protocols for mobile ad hoc networks i.e. periodically the updated routing information is maintained.

Basically OLSR uses multipoint relays (MPRs), a node's one hop neighbor selected for forwarding packets, to reduce the control traffic overhead. OLSR performs better in a large and dense network. Its performance is also better in scenarios where the traffic is random and sporadic between a larger set of node. Following three kinds of control messages are used in OLSR

1) Hello messages are sent periodically to all neighboring node having node's identifier, list of node's neighbors, its MPRs and its neighbors whose bidirectional link have not been yet known.

2) Topology Control (TC) massages are periodically sent by a node having a set of bidirectional links between the node and a subset of node's neighbors. Its purpose is to spread the topological information about the entire network.

3) Multiple Interface Declaration (MID) messages, as indicated by name, are used to declare that a node is running OLSR on multiple interfaces. MPR is responsible for flooding the MID message throughout in the network.

\section{SIMULATION SETUP}

We have used Network Simulator (NS)-2 in our evaluation. The NS-2 is a discrete event driven simulator [5, 6] developed at UC Berkeley. We used Red Hat environment with version NS-2.35 of network simulator NS-2 is suitable for designing new protocols, comparing different protocols and traffic evaluations. It is an object oriented simulation written in $\mathrm{C}++$, with an OTcl interpreter as a frontend. NS uses two languages because simulator got to deal with two things: i) detailed simulation of protocols which require a system programming language which can efficiently manipulate bytes, packet headers and implement algorithms, ii) research involving 
slightly varying parameters or quickly exploring a number of scenarios.

The movement of nodes in the Manhattan Grid Mobility model is generated by a software called Mobility Generator which is based on a frame work called Important (Impact of Mobility Patterns on Routing in Ad-hoc NeTwork, from University of Southern California)[7,17,18].In the scenario we have used three horizontal and three vertical street, fifty nodes can move only on streets and they can turn left or right or move state.

Table 1. Simulation Parameters

\begin{tabular}{|c|c|}
\hline Parameters & Value \\
\hline Routing Protocols & AODV, DSDV, OLSR \\
\hline MAC Layer & 502.11 \\
\hline Packet Size & $1000 \mathrm{~m}^{*} 1000 \mathrm{~m}$ \\
\hline Terrain Size & 50 \\
\hline Nodes & 3 \\
\hline Mobility Model & 3 \\
\hline No. of Horizontal Streets & CBR, TCP \\
\hline No. of Vertical Streets & 10,40 \\
\hline Data Traffic & 900 sec. \\
\hline No. of Source & $0-60 \mathrm{~m} / \mathrm{sec}$ (interval of 10) \\
\hline Simulation Time & \\
\hline Maximum Speed & \\
\hline
\end{tabular}

We have used four traffic patterns with varying number of sources for each type of traffic (TCP and CBR). The sourcedestination pair may be in same street or in different street. The goal of our simulation is to evaluate the performance differences of the three AODV, DSDV and OLSR routing protocols. The type of traffic (CBR and TCP) and the maximum number of sources are generated by inbuilt tool of NS2 [6]. The parameters used for carrying out simulation are summarized in the table 1 .

\subsection{Performance Metrics}

RFC2501 [12] describe a number of quantitative metrics that can be used for evaluating the performance of MANET routing protocols. We have used the following metrics for evaluating the performance of routing protocols (AODV, DSDV \& OLSR):

\subsubsection{Packet delivery ratio:}

It is the ratio of data packets delivered to the destination to those generated by the sources. It is calculated by dividing the number of packet received by destination through the number packet originated from source.

$$
\mathrm{PDF}=(\mathrm{Pr} / \mathrm{Ps})^{*} 100
$$

Where Pr is total Packet received \& Ps is the total Packet sent.

\subsubsection{Routing overhead:}

It is the total number of control or routing (RTR) packets generated by routing protocol during the simulation. All packets sent or forwarded at network layer is consider routing overhead.
Overhead $=$ number of RTR packets

\subsubsection{Normalized routing overhead:}

Number of routing packets "transmitted" per data packet "delivered" at destination. Each hop-wise transmission of a routing is counted as one transmission. It is the sum of all control packet sent by all node in network to discover and maintain route.

$$
\text { NRL }=\text { Routing Packet/Received Packets }
$$

\subsubsection{Average End-to-End Delay (second):}

This includes all possible delay caused by buffering during route discovery latency, queuing at the interface queue, retransmission delay at the MAC, propagation and transfer time. It is defined as the time taken for a data packet to be transmitted across an MANET from source to destination.

$$
\mathrm{D}=(\mathrm{Tr}-\mathrm{Ts})
$$

Where $\operatorname{Tr}$ is receive Time and $\mathrm{Ts}$ is sent Time

\subsubsection{Packet Loss:}

It occurs when one or more packets fail to reach to their destination.

$$
\text { Packet Loss \% }=(1-\mathrm{Pr} / \mathrm{Ps}) * 100
$$

Where Pr is total number of Received Packets and Ps is total number of Sent Packets.

\subsubsection{Throughput (packet/second:}

It is the rate at which network send or receive data. It rated in term of bits or packets per seconds. It is the sum of data rates that are delivered to all nodes in MANET.

$$
\text { Throughput }=\operatorname{Pr} / \mathrm{Pf}
$$

Where Pr is the total number of Received Packets and Pf is the total number of Forwarded Packets.

\section{RESULT AND DISCUSSION}

\section{Packet delivery ratio:}

In case of CBR traffic, AODV deliver almost 93\% packets when network load is low (say 10) and speed is also low. But packet delivery fraction decreases when speed is increased. DSDV delivers almost (45-70\%) originated packets under low network load. OLSR performs better, when speed is low and performance gets degraded when the speed is increasing. When the network load is high (say 40), the packet delivery fraction of all the considered routing protocols is in the region $15-30+\%$ as the node speed increases. AODV performs better than other two considered protocols in all assumed conditions (fig 2). OLSR and DSDV perform better than AODV under TCP traffic source when the network load is high and low (fig $3)$. OLSR is better choice in TCP traffic as shown in figure 3 . Hence we can infer that the overall packet delivery fraction is high (93-99\%) using TCP traffic as compared CBR traffic $(15-93 \%)$ as the speed and network load is increased (low $\rightarrow$ high).

\section{Routing Overhead:}

Routing overhead of AODV under CBR traffic for high and low network load is increasing with the increases in speed. The routing overhead of OLSR and DSDV remain almost constant with the increase in speed and network load. DSDV has the lowest routing overhead under CBR traffic (figure 4). Further it has been observed that routing overhead of DSDV using TCP traffic is also minimum as shown in figure 5 . We can infer that DSDV has minimum routing overhead under CBR and TCP traffics among the considered routing 
protocols. Hence over all routing overhead under TCP traffic is low as compared to CBR traffic. Further it has been observed that with the increase in speed and network load, routing overheads under CBR traffic increases by 16 times as compared to 6 times under TCP traffic for considered protocols under all assumed conditions.

\section{Normalized Routing Overhead:}

In case of CBR traffic, DSDV performs better than AODV and OLSR under low and high network load conditions (figure 6). In case of TCP traffic, DSDV performs better than AODV and OLSR under all assumed conditions (fig 7). Over all, DSDV gives better performance for normalized routing load in both types of traffics and NRL under CBR traffic is high as compared to TCP traffic.

\section{CBR Traffic Sources}

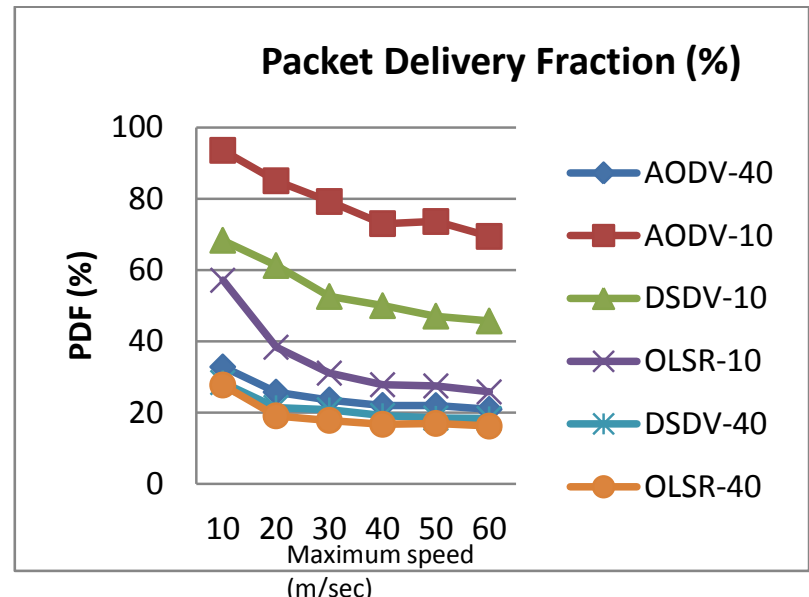

Figure 2: PDF vs Speed

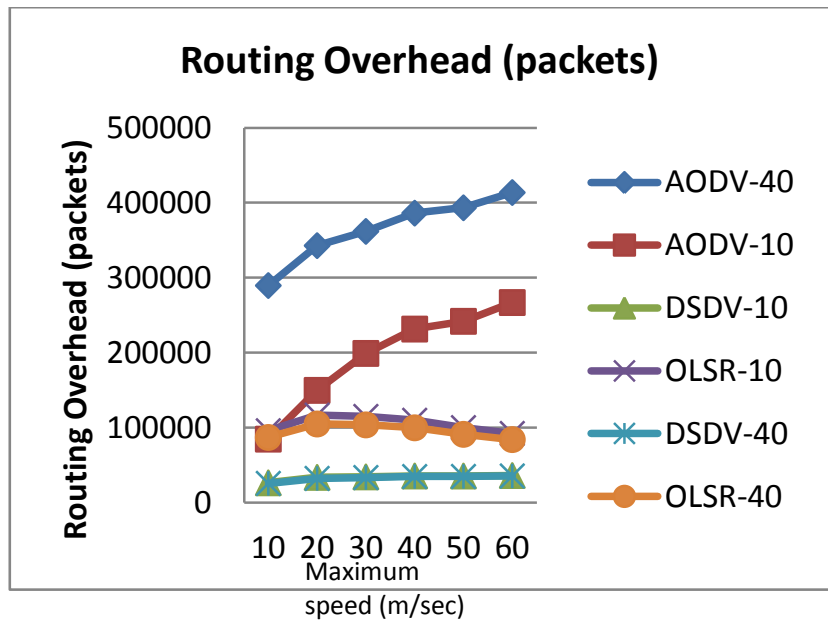

Figure 4: Routing Overhead vs Speed

\section{Average end-to-end Delay:}

In CBR traffic, average end to end delay of AODV is low as compared to DSDV and OLSR under high and low networks load. DSDV give highest delay as compared to other two protocols (figure 8). In case of TCP traffic, AODV gives better result than DSDV and OLSR in high and low network load with enhancement in the speed (fig 9). Over all, AODV is better choice for real time delivery of packet in both types of traffics. The average delay in TCP traffic is low as compared to CBR traffic.

\section{Packet Loss:}

Under CBR traffic, percentage packet loss is less for AODV under high or low networks load as compared to DSDV and OLSR. This is because when link fails, a routing error is passed back to a transmitting node and process repeat. DSDV is also as good as AODV, if packet loss is an indicator.

But packet loss of OLSR is high as compared to other considered routing protocols.

For TCP traffic, the percentage packet loss of AODV is high as compared to other considered routing protocols. In TCP OLSR gives better result than other protocols (fig 11). Over all packet loss rate is low in TCP traffic as compared to CBR traffic.

\section{Throughput:}

DSDV gives highest throughput under high and low network load using CBR traffic sources (fig 12). OLSR gives better throughput than AODV and DSDV under high and low network load using TCP traffic sources (figure 13). Overall, throughput using TCP traffic for all considered routing protocols is much better than CBR traffic.

\section{TCP Traffic Sources}

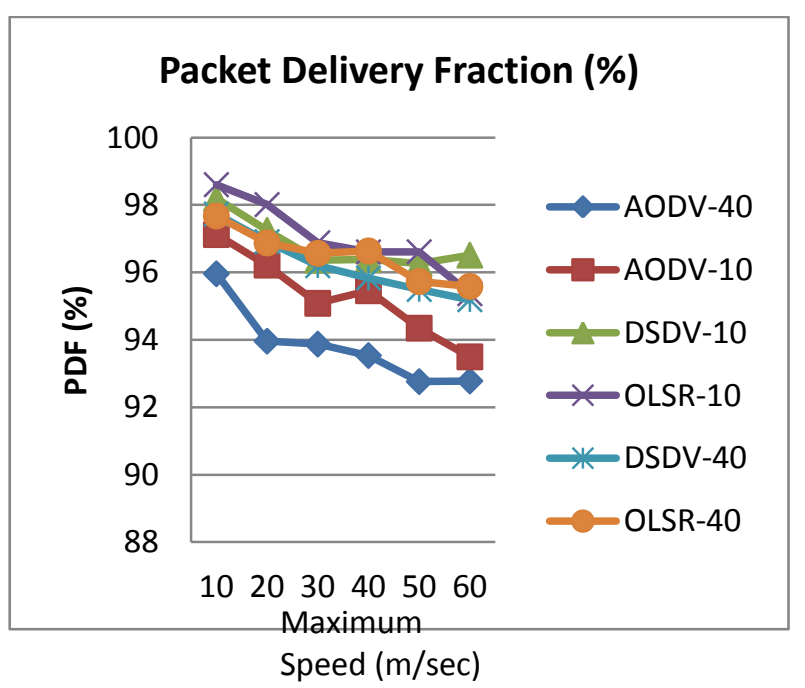

Figure 3: PDF vs Speed

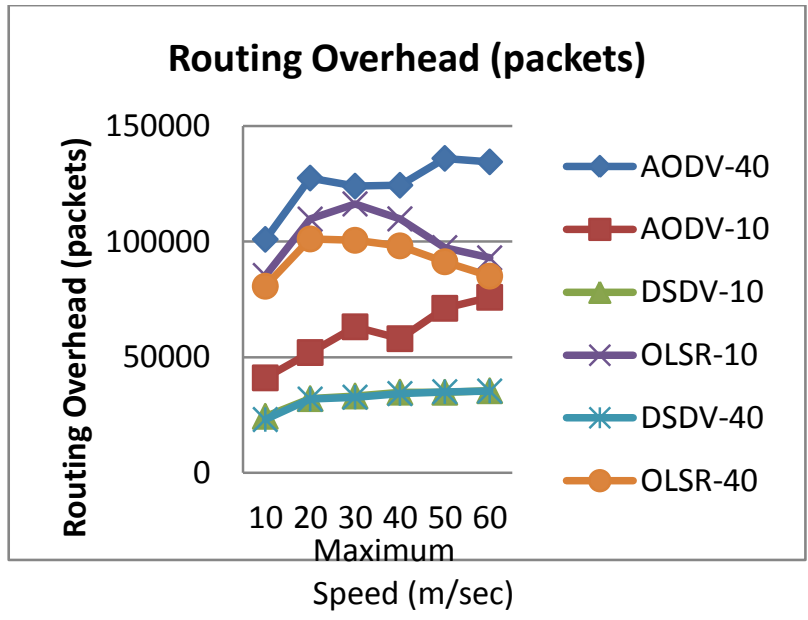

Figure 5: Routing Overhead vs Speed

CBR Traffic Sources 


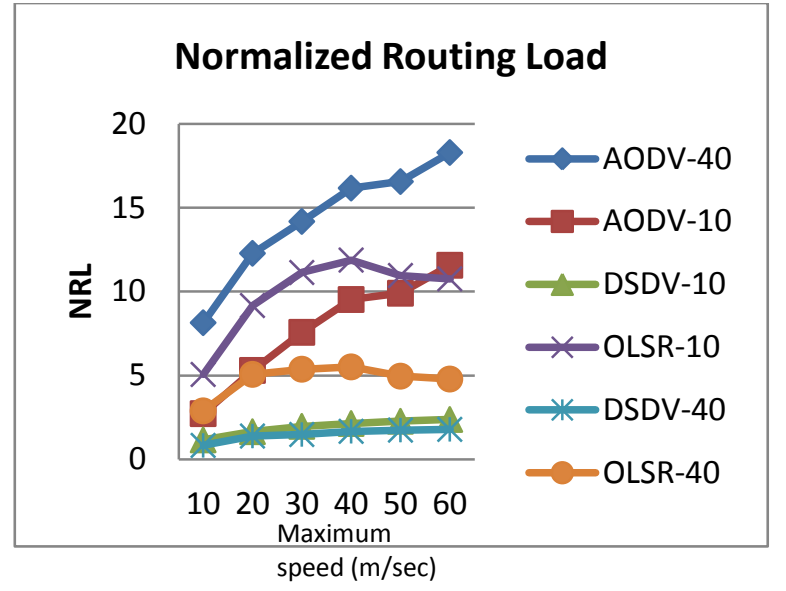

Figure 6: NRL vs Speed

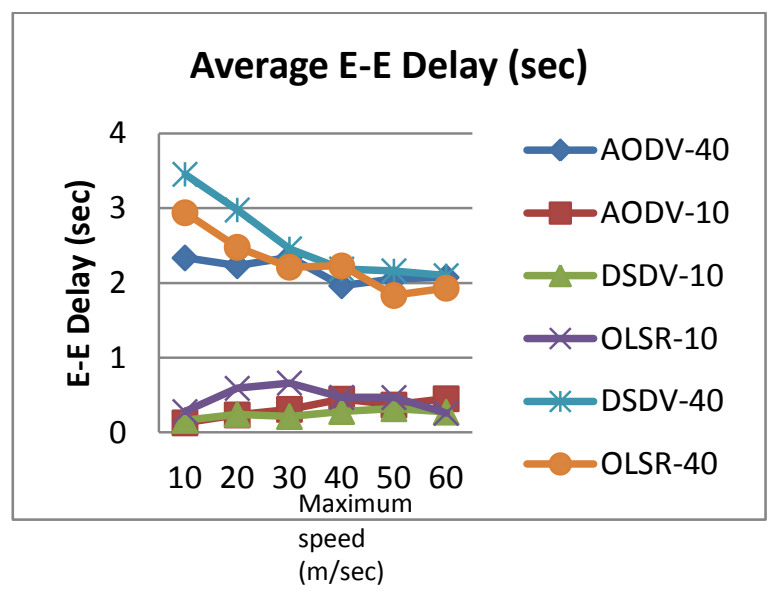

Figure 8:Avg. E-E Delay vs Speed

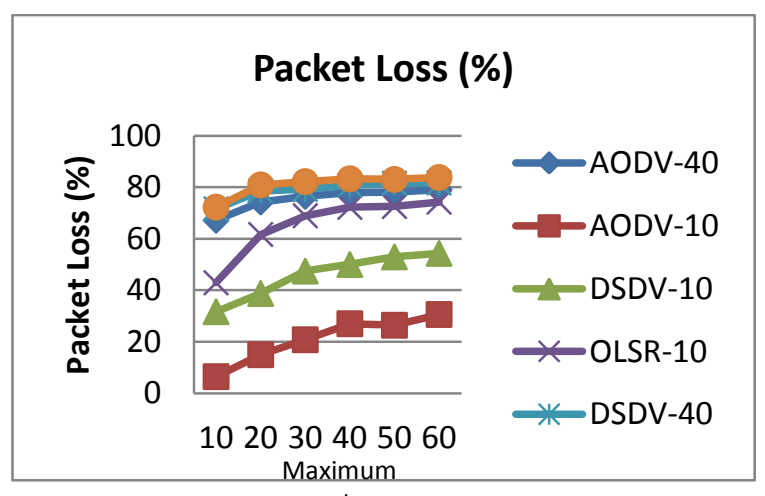

Figure 10: Packet Loss vs Speed

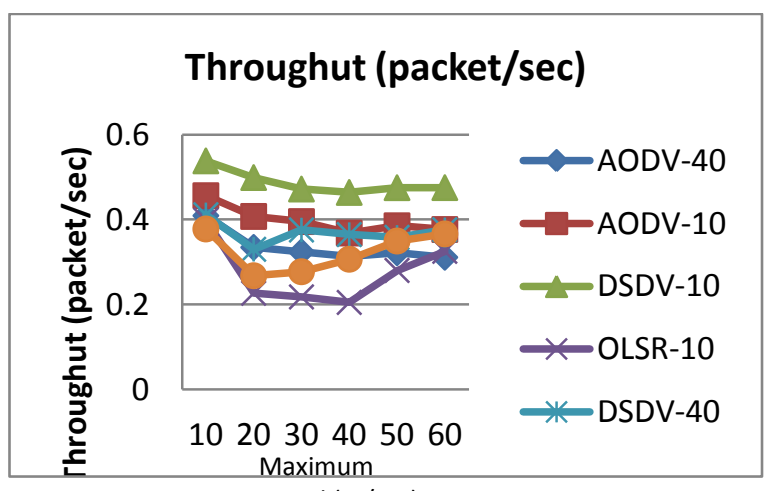

Figure 12: Throughput vs Speed

TCP Traffic Sources

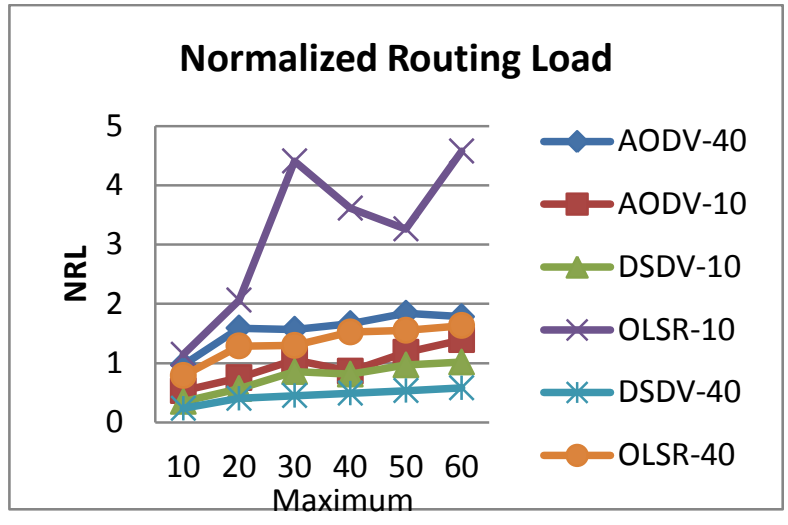

Figure 7: NRL vs Speed

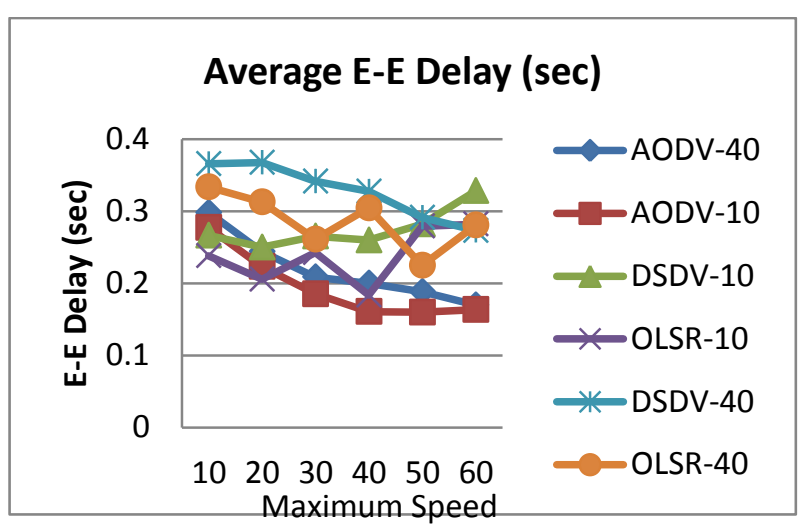

Figure 9: Avg. E-E delay vs Speed

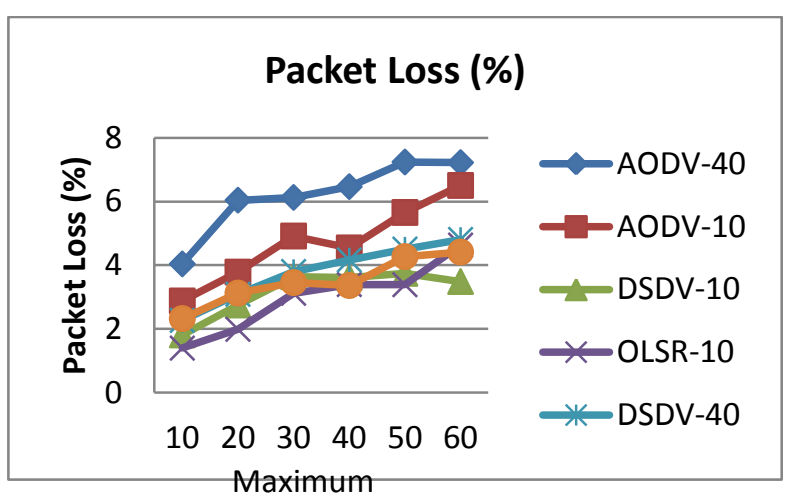

Figure 11: Packet Loss vs Speed

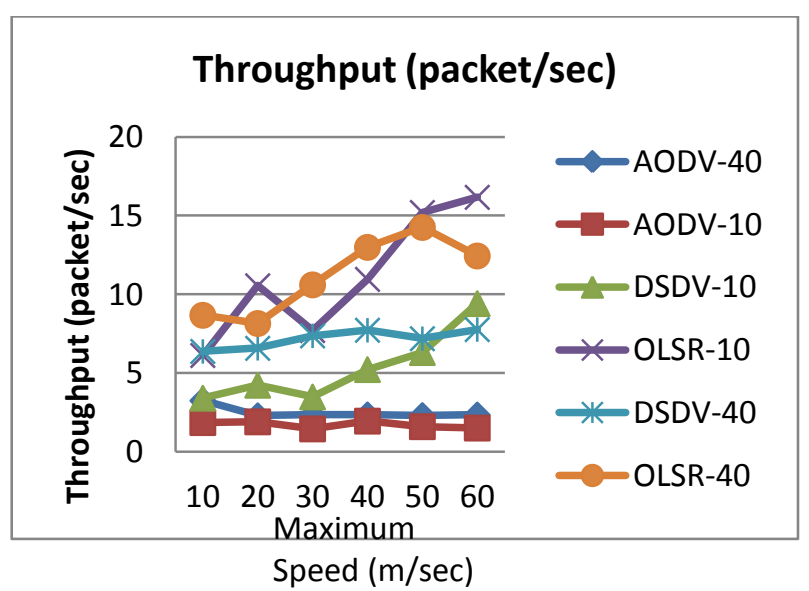

Figure 13: Throughput vs Speed 


\section{CONCLUSION}

From the figures 2-13, we conclude that in Manhattan Grid Mobility Model with CBR traffic sources AODV performs better than OLSR and DSDV at the cost of higher routing overheads. Routing overhead of DSDV is always less than AODV and OLSR. Further AODV is also better choice for real time delivery of packets. Using TCP traffic sources, OLSR gives better results than AODV but at higher routing overheads. Average delay of AODV is less for CBR and TCP traffic. The throughput of OLSR is better in TCP traffic. Hence all the considered routing protocols (AODV, DSDV and OLSR) perform better under TCP traffic as compared to CBR traffic.

In this paper, we have investigated the performance of only three routing protocol using CBR and TCP traffic under Manhattan Grid Mobility Model. Further study could be conducted on the other MANET routing protocols under different mobility model and using different types of traffic sources.

\section{REFERENCES}

[1] S. Das, C. E. Perkins, E. Royer, "Ad Hoc On Demand Distance Vector (AODV) Routing", IETF Draft, June 2002.

[2] C-K Toh "Ad Hoc Mobile Wireless Networks Protocols and Systems", First Edition, Prentice Hall Inc, USA, 2002.

[3] C.E. Perkins and E.M.Royer, "Ad-Hoc On Demand Distance Vector Routing", Proceedings of the 2nd IEEE Workshop on Mobile Computing Systems and Applications, New Orleans, LA, USA, pages 90-100, February 1999.

[4] Elizabeth M. Royer and Chai-Keong Toh, "A Review of Current Routing Protocols for Ad Hoc Mobile Wireless Networks", IEEE Personal Communications, pages 4655, April 1999.

[5] UCB/LBNL/VINT Network Simulator, http://wwwmash. cs.berkeley.edu/ns/, referred on January 2015.

[6] "The Network Simulator - ns-2," available at http://www.isi.edu/nsnam/ns/ , referred on January 2015.

[7] Fan Bai, Ahmed Helmy "A Framework to systematically analyze the Impact of Mobility on Performance of Routing Protocols for Adhoc Networks", IEEE INFOCOM 2003.

[8] Tracy Camp, Jeff Boleng, Vanessa Davies "A Survey of Mobility Models for Ad Hoc Network Research", Wireless Communication \& Mobile Computing (WCMC): vol. 2, no. 5, pp. 483-502, 2002.
[9] Perkins Charles E, Bhagwat Pravin, "Highly Dynamic Destination-Sequenced Distance Vector Routing (DSDV) for Mobile Computers", In SIGCOMM UK, pp. 234-244, 1994

[10] Suresh Kumar, R.K. Rathy and Diwakar Pandey, "Traffic Pattern Based Performance Comparison of Two Reactive Routing Protocols for Ad-hoc Networks using NS2", 2nd IEEE International Conference on Computer Science and Information Technology, 2009.

[11] T.H. Clausen and P. Jacquet, "Optimized Link State Routing (OLSR)”, RFC 3626, October 2003.

[12] S.Corson and J.Macker, "Routing Protocol Performance Issues and Evaluation considerations", RFC2501, IETF Network Working Group, January 1999.

[13] S. R. Biradar, Hiren H D Sharma, Kalpana Shrama and Subir Kumar Sarkar, "Performance Comparison of Reactive Routing Protocols of MANETs using Group Mobility Model", IEEE International Conference on Signal Processing Systems, pages 1921952011.

[14] C. Perkins, E. Belding-Royer, S. Das, quet, "Ad hoc OnDemand Distance Vector (AODV) Routing", RFC 3561, July 2003

[15] N.Aschenbruck,E.Gerhands-Padilla, ,P.Martini,”A Survey on mobility models for Performance analysis in Tactical Mobile networks," Journal of Telecommunication and Information Technology,Vol.2 pp.54-61,2011.

[16] X. Hong, M. Gerla, G. Pei, and C.-C. Chiang, "A group mobility model for ad hoc wireless networks," in ACM/IEEE MSWiM, August 1999.

[17] http://www-scf.usc.edu/ fbai/important/, referred on February 2015

[18] http://nile.usc.edu/important/, referred on February 2015.

[19] G. Jayakumar and G. Gopinath "Performance Comparison of MANET Protocols Based on Manhattan Grid Mobility Model", Journal of Mobile Communication 2(1) 18-26, 2008

[20] Nor Surayati Mohamad Usop, Azizol Abdula and Ahmad "Performance Evaluation of AODV, DSDV \& DSR Routing Protocol in Grid Environment", IJCSNS International Journal of Computer Science and Network Security, VOL.9 No.7, July 2012. 